\title{
Research of lithium capillary-pore systems for fusion reactor plasma facing components
}

\author{
V.A. Evtikhin ${ }^{\text {a,* }}$, A.V. Vertkov ${ }^{\text {a }}$, I.E. Lyublinski ${ }^{\text {a }}$, B.I. Khripunov ${ }^{\text {b }}$, \\ V.B. Petrov ${ }^{b}$, S.V. Mirnov ${ }^{\text {c }}$ \\ a State Enterprise 'Red Star', Electrolitnij Proezd 1a., 115230 Moscow, Russia \\ ${ }^{\mathrm{b}}$ Nuclear Fusion Institute, RRC Kurchatov Institute, Moscow 123182, Russia \\ ${ }^{\mathrm{c}}$ Troitsk Institute for Innovative and Fusion Research, Moscow Region 142190, Russia
}

\begin{abstract}
To date there is no adequate solution for high heat load plasma facing components of the next step fusion reactor among solid material options. A lithium-filled capillary porous systems (CPS) was proposed as a plasma facing material and experimental work on this subject is now in progress. Steady-state experiments with CPS-based target and lithium supply systems have shown successful operation at heat fluxes of $1-10 \mathrm{MW} / \mathrm{m}^{2}$ during several hours. Experimental data is obtained on lithium CPS stability at heat flux up to $25-50 \mathrm{MW} / \mathrm{m}^{2}$. The lithium CPS behaviour in contact with real tokamak plasma is considered for normal discharge condition at $10 \mathrm{MW} / \mathrm{m}^{2}$ and for plasma disruption at $15 \mathrm{MJ} / \mathrm{m}^{2}$. Erosion mechanism of lithium under tokamak plasma impact was analysed. Stability of lithium CPS in tokamak conditions was shown.
\end{abstract}

(C) 2002 Elsevier Science B.V. All rights reserved.

\section{Introduction}

The ITER project development showed that conventional technical solutions of divertor and divertor plates with low $Z$ solid material for plasma burning practically in steady-state in a tokamak of such a scale met with serious difficulties. An idea to use liquid metals as plasma facing materials in fusion reactors with magnetic and inertial confinement has attracted attention for a long time to control high heat and particle loads [1-4].

A new idea of liquid metals application in tokamaks was advanced on the basis of the surface tension forces in capillary channels. These capillary channels may be realized in the form of so-called capillary-pore systems (CPS) [5-7]. Self-regeneration is an intrinsic property of

\footnotetext{
${ }^{*}$ Corresponding author. Tel.: +7-095 978 4443; fax: +7-095 2845843. hin).

E-mail address: evtikhin@protein.bio.msu.ru (V.A. Evtik-
}

such a structure in contact with plasma. This property becomes essentially important if we take into account that the ITER divertor plate will operate in presence of frequent small disruptions - ELMs - that are the reason of an enhanced erosion. One may expect that surface self-regeneration will become the most important factor for the reactors next to ITER-FEAT.

Lithium compatibility with the tokamak plasma becomes a special issue being now intensively studied. It is divided into two parts: character of lithium influx from the wall and lithium behaviour in the plasma. Some of these concerns may be clarified at present, first, on the basis of positive experience of lithium injection into the hot plasma of tokamak TFTR [8,9] and of T-11M tokamak operation experience with liquid lithium CPS limiter $[10,11]$. The divertor concept which is based on the lithium CPS application has been presented [11].

We propose to use CPS and by this to realize all advantages of liquid lithium as plasma facing material. We consider different aspects of this proposal taking into account the gained experience and we present the results of simulating experiments. 


\section{Lithium capillary-pore systems}

The following basic considerations were assumed as a starting point [5-7,11-15]. A natural way to make a safe divertor target plate and other plasma facing elements is a decrease in power load through its redistribution over a larger area by evaporation-condensation mechanism and by radiation. In this case heat removal may be done with the highest performance with an appropriate choice of the working fluid (for lithium it is hundreds of $\mathrm{MW} / \mathrm{m}^{2}$ at temperatures below the boiling point) and can be controlled by temperature conditions. Finally, power removal from the divertor will be carried out without overloads by the thermal conductivity to cooling loops and further to the energy conversion system.

CPS is used in the target plate design to confine liquid metal on a surface of any configuration and orientation with no cavities in lithium film and in absence of noncontrolled surface flows due to capillary forces and to feed the evaporating surface with liquid metal. Moreover, there is no effect of lithium film separation from the surface and baring of CPS solid structure under plasma impact. Characteristics of CPS (changing porosity, anisotropic permeation, working surface geometry etc.) may be varied in a large range corresponding to fabrication technology. CPS structure ensures sufficient pressure of working fluid in the feeding system just due to capillary pressure. The system is self-sustaining and self-regenerating because the CPS working fluid pressure distribution is extremely sensitive to changes in local heat load distribution on its surface. The same principle is realized in heat pipes of different applications in practice.

Application of lithium CPS makes the proposed divertor concept highly efficient and this has a number of principally new features so that it appears to be practically feasible for the following reasons: lithium has a low $Z$ that determines minimal disturbance of the main plasma; lithium evaporation, radiation and ionization lead to redistribution of the important part of coming energy, thus decreasing power load density on the divertor; lithium corresponds well to reactor design with self-cooled lithium-lithium blanket; service systems will be common both for blanket and divertor; tritium extraction technology may be taken the same for both components; the same structure material may be used in those systems - low activated vanadium alloys that are compatible well with lithium at temperatures below 700 ${ }^{\circ} \mathrm{C}$; the tritium accumulation in the target elements of the divertor may be controlled, the tritium content may be maintained at a required level in the circulating liquid metal; lithium vapour may be easily condensed in contrast to gases; the condensed liquid metal will come from condensation zone to circulation system and will not be accumulated in the divertor and around it as dust unlike the solid material divertor; low speed of lithium flow and insulating self-healing coatings $[13,16]$ will efficiently reduce MHD effects.

Long service life of target elements will be ensured by the following properties:

- erosion of the target plate is mainly caused by lithium evaporation, sputtering and splashing and it is compensated due to constant feeding with liquid lithium;

- thermal gradients will not give rise to stresses in the lithium-filled CPS because no thermal stress occurs in liquid lithium; estimated maximal heat flux for CPS is more than $10^{3}$ times higher than that for monolithic solid material; consequently, no cracking and no fatigue cracks will occur on the target plates unlike the solid divertor surface;

- the problem of radiation resistance practically does not arise for CPS.

Different solid materials may serve as CPS basis metal wire mesh, metal felt, sintered powders etc. The choice of CPS structure material is determined by operating conditions according to the requirements here below: low sensibility of mechanical properties to radiation damages; high resistance to high temperature gradients; ability to keep properties in condition of partial damage; compatibility with liquid metal; good wetting with liquid metal; acceptable fabrication technology of structure elements.

To our mind, according to the available data metal ( $\mathrm{V}$ and Mo alloys, stainless steels etc.) would be the best CPS structure materials. Fibre-based systems, namely, metal meshes and felts, which correspond to this requirements, have well-developed fabrication technology and are not expensive. So lithium CPS may be considered as a new class of plasma facing material with unique properties which correspond to numerous and contradictory requirements.

Liquid metal divertor CPS operation efficiency will depend on its design, on solid structure wetting with lithium, on capillary pressure.

Self-regulation is an intrinsic property of CPS. It is based on the dependence of capillary forces on the meniscus radius $R\left(R_{\mathrm{eff}}, Q\right)$ at the evaporating surface depending, in turn, on pore radius $R_{\text {eff }}$ and on incident power flux $Q[13,17]$. Capillary pressure is defined by the following expression:

$P_{\mathrm{c}}=2 \sigma(T) \cos \theta / R\left(R_{\mathrm{eff}}, Q\right)$,

where $\sigma(T)$ is lithium surface tension, $\theta$ is the edge wetting angle. Capillary pressure in CPS at full wetting and acceptable pore size reach $\simeq 0.1-0.5 \mathrm{MPa}$.

Lithium confinement and damage resistance of CPS in stationary conditions are defined by the following inequality for all points of the evaporation surface: 
$P_{\mathrm{c}} \geqslant \Delta P_{\mathrm{t}}+\Delta P_{\mathrm{f}}+\Delta P_{\mathrm{m}}+\Delta P_{\mathrm{h}}+P_{0}+P_{\mathrm{p}}$

where $\Delta P_{\mathrm{t}}$ is the liquid-vapour phase transition pressure difference, $\Delta P_{\mathrm{f}}$ is the hydrodynamic pressure loss of lithium flow in CPS, $\Delta P_{\mathrm{m}}$ is the MHD pressure loss in CPS in magnetic field, $\Delta P_{\mathrm{h}}$ is the hydrostatic pressure drop in CPS, $P_{0}$ is the pressure in the supply system, $P_{\mathrm{p}}$ is the pressure of incident plasma on the CPS surface.

\section{Stability of the lithium CPS under stationary and pulsed power loads}

\subsection{Experimental study of lithium CPS in stationary conditions}

CPS-based mock-ups of lithium targets have been tested under stationary high power heat load to prove the possibility of CPS application as plasma facing material. Experiments have been performed in the linear beamplasma simulator SPRUT-4 [13] providing power flux of 1-200 MW/m². Balance of lithium mass and energy balance were studied in the range of $1-25 \mathrm{MW} / \mathrm{m}^{2}$. Different target modifications have been tested. The latest modification ensured stabilization of thermal conditions in the target by forced water cooling [18].

The experiments have shown $[13,18,19]$ that a simple model of lithium CPS target $\left(S=3 \mathrm{~cm}^{2}\right)$ can carry long heat loads up to $25 \mathrm{MW} / \mathrm{m}^{2}$ and short excursions to 50 $\mathrm{MW} / \mathrm{m}^{2}$ (Fig. 1). Further experiments have been carried out with liquid lithium open loop providing sufficient lithium supply in stable thermal conditions in the target during long irradiation tests. This target modification has been shown to operate in steady-state during a long time period (up to $3 \mathrm{~h}$ ) at power flux from 1 to $10 \mathrm{MW} /$ $\mathrm{m}^{2}$ [19]. Lithium particle loss rate from the CPS target in

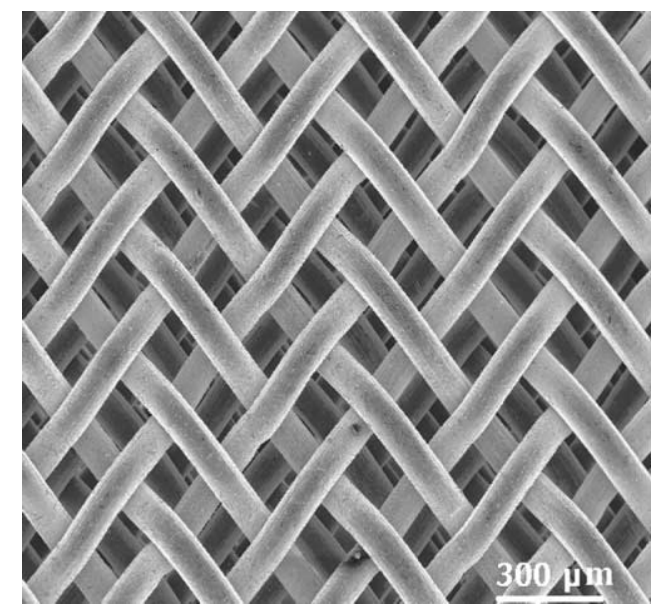

Fig. 1. CPS based on the Mo mesh $\left(R_{\text {eff }}=75 \mu \mathrm{m}, d_{\text {wire }}=100\right.$ $\mu \mathrm{m}$ ) after tests at $10-25 \mathrm{MW} / \mathrm{m}^{2}, 1.5 \mathrm{~h}$ (lithium removed after test). this range of incident power was determined by evaporation process and was equal to $5 \times 10^{19}-2 \times 10^{21}$ $\mathrm{s}^{-1} \mathrm{~cm}^{-2}$. Note that the CPS surface temperature depended on the incident heat flux and laid within the $350-970{ }^{\circ} \mathrm{C}$ range.

Lithium plasma has been generated in front of the target and it propagated along magnetic field to a distance $10-20 \mathrm{~cm}$. Lithium neutral and ion radiation has been studied. No indications of CPS structure material lines (Mo) have been found in the studied spectra. These observations gave evidence of a low sputtering effect in our conditions.

The following processes have been observed in this experiment: lithium loss from the surface was compensated by lithium feed due to surface tension forces, ratio of the removed power by evaporation to incident power achieved 0.7 ; vapour condensation occurred on the wall in the beam transport channel, these processes being intensive enough to provide necessary conditions for electron gun operation without break-down.

The obtained results confirm that lithium target with CPS surface structure can operate efficiently in stationary conditions at high power loads.

\subsection{Disruption simulation experiments}

The effects of disruption discharges in tokamaks have been simulated by magnetized plasma flows interacting with lithium capillary structure in quasi-stationary plasma accelerator QSPA $[12,13,17,20]$. Experimental models of CPS have been tested at disruptive high heat loads. The plasma flow parameters were: plasma density $n_{\mathrm{e}} \approx(2-5) \times 10^{16} \mathrm{~cm}^{-3}$, temperature $T_{\mathrm{e}}+T_{\mathrm{i}} \approx 30$ $\mathrm{eV}$, magnetic field in plasma $B \approx 1 \mathrm{~T}$, energy flux $Q=4$ $5 \mathrm{MJ} / \mathrm{m}^{2}$, pulse duration $\tau=200-500 \mu$ s, diameter of plasma flow $d=40-80 \mathrm{~mm}$, plasma pressure $P \approx$ $4 \times 10^{5} \mathrm{~Pa}$. The lithium-filled CPS was shown to absorb energy under plasma disruptions without failure (Fig. 2).

Two effects were observed to occur during irradiation: shielding layer formation near the target surface and droplet erosion.

\subsection{Shielding layer}

A dense plasma layer was formed in front of the target during interaction. Interferograms of the process have shown that at $5 \mu$ s the plasma density reached $n_{\mathrm{e}}=10^{17} \mathrm{~cm}^{-3}$. Then it decreased and an opaque layer $\delta \approx 10-15 \mathrm{~mm}$ thick was formed in front of the target. Turbulent processes in this layer may explain it. The evaporated neutral lithium appears in about $10 \mu$ s after start-up of the plasma interaction with the target at a distance of about $10 \mathrm{~mm}$ from it. The layer becomes 40 $50 \mathrm{~mm}$ thick by the moment $\tau \approx 200 \mu \mathrm{s}$. The major part of the plasma energy, $\simeq 97-99 \%$, is absorbed and radiated in this layer which plays the role of a shielding 

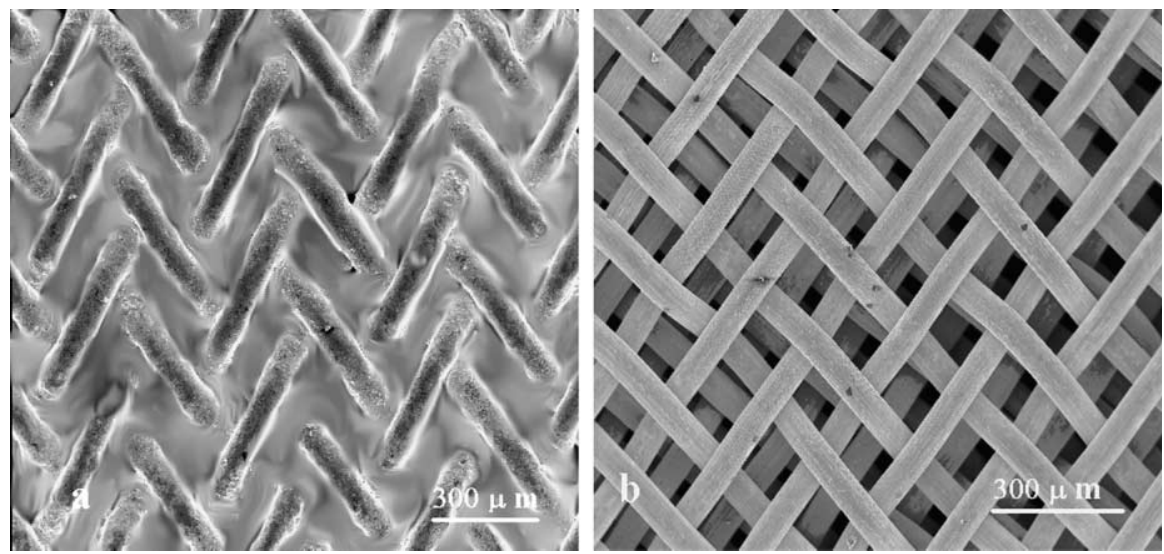

Fig. 2. Surface view of CPS based on the Mo mesh $\left(R_{\mathrm{eff}}=75 \mu \mathrm{m}, d_{\text {wire }}=100 \mu \mathrm{m}\right)$ after tests with 22 plasma shots at disruption condition ( $\left.5 \mathrm{MJ} / \mathrm{m}^{2}, t=500 \mu \mathrm{s}\right)$ : (a) Li filled, (b) Li removed.

layer. A small part of liquid lithium was evaporated from the target after every shot. The target itself remained undamaged even after 22 plasma shots (Fig. 2). By contrast, a special target made of a molybdenum mesh without lithium was destroyed by the plasma flow after a single shot. A similar result has been observed later experimentally in a T-11M tokamak: only $30-50 \mathrm{~J}$ of about $0.7 \mathrm{~kJ}$ of total plasma energy loss has been found to reach the rail limiter during disruption events while under normal discharge condition the part of energy coming to limiter achieved $50 \%$ of the total flow to the wall [21].

\subsection{Droplet erosion}

Lithium erosion by evaporation in the first $5-10 \mu$ s of plasma pulse was just a small fraction of total mass loss $(\simeq 5-10 \mu \mathrm{m})$ including periods of shielding layer development and evolution. Much higher erosion of liquid surface was induced by splashing. Splashing could arise as a result of 'wind waves', Kelvin-Helmholtz hydrodynamic instabilities and volume bubble boiling. Droplet ablation erosion rate was measured for a free lithium surface and it achieved $\simeq 1-3 \mathrm{~mm}$ per pulse at heat fluxes up to $3 \mathrm{GW} / \mathrm{m}^{2}$ that agreed well with estimations. For porous structures, the lithium-eroded layer thickness was considerably suppressed (from 60 to $1 \mu \mathrm{m}$ for decreasing effective pore radius from 200 to 10 $\mu \mathrm{m})$ by capillary forces. No solid structure damage was observed since the lost lithium layer was restored immediately after every shot.

Laser scattering technique was applied to estimate the total amount of droplets and their size distribution. The large size fraction $(0.5-1 \mathrm{~mm})$ depended strictly on the CPS parameters (it increased with pore radius) and on the CPS surface orientation with respect to the inci- dent plasma flow (increased with incident angle deviation from $90^{\circ}$ ). The main particle loss was observed in the surface plane. The droplet expansion velocity was $0.1-10$ $\mathrm{m} / \mathrm{s}$. CPS with initially solid lithium $\left(T<T_{\text {melt }}\right)$ showed an increase of erosion rate for increasing number of pulses. This was not the case for CPS with initially liquid lithium $\left(T>T_{\text {melt }}\right)$. This effect was attributed to wave relief observed on the solid lithium to be formed with increasing number of shots thus causing higher erosion at every next shot compared to initially smooth surface. No waves and relief were formed on the surfaces with $T>T_{\text {melt }}$. This effect proves one of the advantages of CPS with liquid metal in comparison with solid target.

Ablation erosion may be efficiently suppressed by an optimal choice of CPS parameters (pore radius $\approx 10-100$ $\mu \mathrm{m})$ and the conditions may be achieved when the CPS base material is not eroded, damaged or melted.

These results indicate that lithium CPSs have an evident advantage compared to solid targets because they do not practically loose their mass; their geometrical characteristics and capillary properties are well conserved under the studied experimental conditions.

\section{Interaction of plasma with lithium capillary-pore structure in tokamak}

Experiments in the T-11M tokamak have been performed with the main aim to prove the compatibility of a lithium CPS with boundary plasma in tokamak conditions close to quasi-stationary conditions expected in reactors $[10,12,15,18,21,22]$. The first task was to ascertain whether spontaneous lithium bursts from the liquid wall to the chamber volume were an important effect or not. Besides, lithium interaction with working gases, lithium migration in plasma, technology of lithium application in tokamak have been studied. 

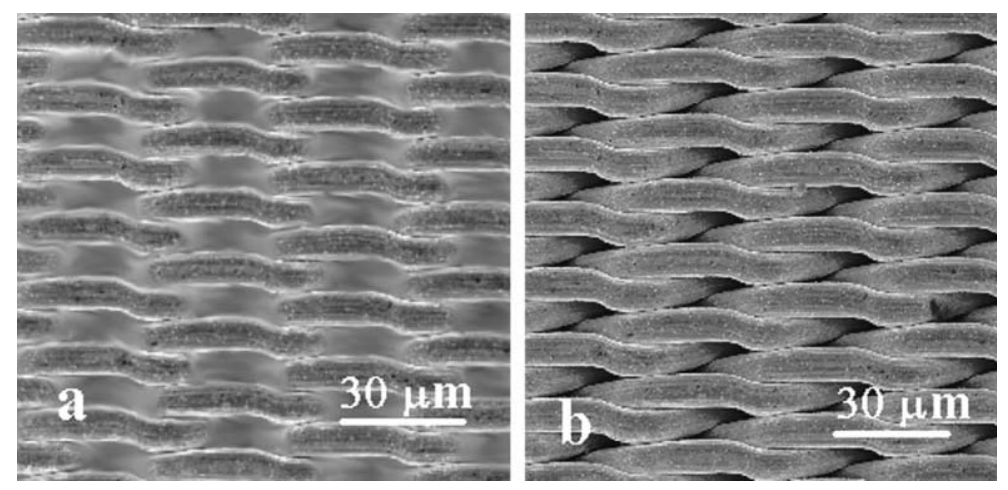

Fig. 3. Surface view of CPS based on the 316-type steel mesh $\left(R_{\text {eff }}=15 \mu \mathrm{m}, d_{\text {wire }}=10 \mu \mathrm{m}\right)$ after test with 2000 plasma shots in T-11M tokamak (10 MW/m², $\tau=0.1 \mathrm{~s})$ : (a) Li filled, (b) Li removed.

The performance data of the small tokamak device T-11M are the following: $R=0.7 \mathrm{~m}, a=0.2 \mathrm{~m}, B_{\mathrm{t}}=$ $1 \mathrm{~T}$, plasma current $J_{\mathrm{p}} \approx 100 \mathrm{kA}$, discharge pulse length about $0.1 \mathrm{~s}$, boundary plasma temperatures $T_{\mathrm{e}}=20-30$ $\mathrm{eV}$. Heat flux to limiter is about $10 \mathrm{MW} / \mathrm{m}^{2}$. Movable rail limiter with plasma contacting surface made of lithium CPS (two versions of CPS were studied with $R_{\text {eff }}=100$ and $15 \mu \mathrm{m}$ ) was inserted into plasma to about $5 \mathrm{~cm}$ thus limiting plasma column aperture and determining plasma current $(q(a)=3-4)$.

The study of the first limiter design showed that ponderomotive forces applied to the edges of it in disruption conditions were underestimated. As a result, splashing of lithium in the direction of field lines was observed. This effect was suppressed with the second limiter design $\left(R_{\text {eff }}=15 \mu \mathrm{m}\right)$ where the confinement condition similar to (2) was satisfied with a good margin.

No catastrophic events leading to lithium injection in MHD stable discharge conditions within the whole lithium temperature range (from 20 to $600{ }^{\circ} \mathrm{C}$ ) have been observed in T-11M experiments and it was the first important result of the work. Lithium and graphite limiters worked practically in a similar way if an additional heater was not used $[10,15]$. Heating of the lithium limiter gave rise to lithium injection into the plasma detected by an increase of lithium line radiation and of integral light emission in the vicinity of the limiter. Estimations of absolute lithium emission have shown [22] that for limiter temperatures $T_{0}<500{ }^{\circ} \mathrm{C}$ it remains in the limits expected for sputtering by $\mathrm{D}^{+}$and $\mathrm{Li}^{+}$ions with sputtering yield from 0.5 to 1 . This is in correlation with the known data on sputtering [23]. The rise of lithium flux during discharge for $T_{0}>200{ }^{\circ} \mathrm{C}$ may be attributed to self-sputtering by $\mathrm{Li}^{+}$ions accumulated in the plasma periphery. For temperatures higher than $T_{0} \approx 500{ }^{\circ} \mathrm{C}$ evaporation appears to become the main channel of lithium emission.

Therefore, lithium emission into discharge could be controlled by an increase of the initial limiter tempera- ture in T-11M. One could expect to obtain a growth of periphery radiation and, by this, to reduce the heat load to the limiter. It was really reduced by approximately a factor of two by these manipulations in the helium discharge $[10,15]$.

The obtained results confirm that lithium CPS can operate efficiently in real tokamak conditions (Fig. 3).

\section{Conclusion}

The use of liquid lithium filled CPS as a plasma facing material in a tokamak has a high potential. First, lithium injected into the plasma periphery even in considerable quantity does not cause catastrophic consequences for a plasma column.

The surface tension forces may be used to form a free liquid metal surface with CPS thus solving the problem of ponderomitive forces applied to the chamber and to divertor surface layers, i.e. the problem of sufficient confinement of liquid metal and regeneration of its surface in contact with the plasma.

A complex of experimental studies has been carried out with CPS having different characteristics (effective pore radius, material etc.) under high stationary (up to $25 \mathrm{MW} / \mathrm{m}^{2}$ ) and under high power plasma pulses. Their function without damages has been demonstrated in these experiments. Proceeding from these results a long service life of wall elements designed on their basis in stationary and disruption conditions may be predicted.

A series of experiments on the T-11M tokamak has proven the compatibility of the lithium CPS limiter with the plasma in all operating conditions. No spontaneous lithium bursts at a heat load close to that of reactor level 10 of $\mathrm{MW} / \mathrm{m}^{2}$ has been observed. High energy loss by lithium radiation has been detected including the case of disruption events so that a solid basis of CPS limiter had no damage after more than $2 \times 10^{3}$ plasma shots. A number of unique properties of lithium determines its 
high potential for application for heat removal at the plasma-wall boundary.

\section{References}

[1] G.L. Kulcinski, J. Nucl. Mater. 85\&86 (1979) 87.

[2] V.G. Karasev et al., Proceedings of 4th IAEA TCM on fusion reactor design and technology, vol. 2, Yalta, 26 May-6 June 1986, IAEA-TC-392.3/51, IAEA, Vienna, 1987, p. 239.

[3] UWMAK-I, A Wisconsin Toroidal Fusion Reactor Design Study, UWFDM-68, University of Wisconsin, 1974.

[4] I.R. Kirillov, E.V. Muraviev, in: Fusion Technology 1996, Elsevier Science B.V., 1997, p. 251.

[5] V.A. Evtikhin, L.G. Golubchikov, Divertor of fusion reactor, RF Patent 2051430, 7 February 1995.

[6] V.A. Evtikhin, I.E. Lyublinski, A.V. Vertkov, et al., Fusion Energy, vol. 3, IAEA, Vienna, 1997, p. 659.

[7] L.G. Golubchikov, V.A. Evtikhin, I.E. Lyublinski, et al., J. Nucl. Mater. 233-237 (1996) 667.

[8] C.H. Skinner et al., J. Nucl. Mater. 241-243 (1997) 214.

[9] D.K. Mansfield, Overview of TFTR Li experiments, SNL, Albuquerque NM, 13-17 November 2000.

[10] V.B. Lazarev, E.A. Azizov, S.V. Mirnov, et al., Proceedings of 26th EPS Conference on Controlled Fusion Plasma Physics, ECA, vol. 231, 1999, p. 845.

[11] V.A. Evtikhin, I.E. Lyublinski, A.V. Vertkov, et al., Proceedings of the IAEA/TCM on Divertor Concepts, Aix en Provence (France), 11-14 September 2001, \#029, Plasma Phys. Control. Fusion 44 (2002) 955.

[12] V.A. Evtikhin, I.E. Lyublinski, A.V. Vertkov, et al., Proceedings of the 17 th International Conference on
Fusion Energy, Yokohama, Japan, 19-24 October 1998, Fusion energy, vol. 4, 1998, IAEA, Vienna, 1999, IAEACSP-1/P, p. 1309.

[13] V.N. Mikhailov, V.A. Evtikhin, I.E. Lyublinski, et al., Lithium for Fusion Reactors and Space Nuclear Power Systems of XXI Century, Energoatomizdat, Moscow, 1999.

[14] V.A. Evtikhin, I.E. Lyublinski, A.V. Vertkov, et al., Proceedings of the 18th International Conference on Fusion Energy, Sorrento, Italy, 4-10 October 2000, IAEA-CSP-8/C, an IAEA CD-ROM, FTP1/24, IAEA, 2001.

[15] V.A. Evtikhin, I.E. Lyublinski, A.V. Vertkov, et al., Proceedings of the 18th International Conference on Fusion Energy, Sorrento, Italy, 4-10 October 2000, IAEA-CSP-8/C, an IAEA CD-ROM, EXP4/21, IAEA, 2001.

[16] A.V. Vertkov, V.A. Evtikhin, I.E. Lyublinski, Fus. Eng. Des. 58\&59 (2001) 731.

[17] V.A. Evtikhin, I.E. Lyublinski, A.V. Vertkov, et al., J. Nucl. Mater. 271\&272 (1999) 396.

[18] V.A. Evtikhin, I.E. Lyublinski, A.V. Vertkov, et al., Fus. Eng. Des. 49\&50 (2000) 195.

[19] B.I. Khripunov, V.B. Petrov, V.V. Shapkin, et al., J. Nucl. Mater. 290-293 (2001) 201.

[20] N.V. Antonov, V.G. Belan, V.A. Evtikhin, et al., J. Nucl. Mater. 241-243 (1997) 1190.

[21] A.M. Belov, V.B. Lazarev, A.G. Alekseev, et al., in: Proceedings of the 28 EPS Conference on Controlled Fusion and Plasma Physics, Madeira, 2001, P5. 108.

[22] V.A. Evtikhin, I.E. Lyublinski, A.V. Vertkov, et al., Fus. Eng. Des. 56\&57 (2001) 363.

[23] J.P. Allain, D.N. Ruzic, M.R. Hendricks, J. Nucl. Mater. 290-293 (2001) 180. 